\title{
Domain walls of single-component Bose-Einstein condensates in external potentials
}

\author{
P.G. Kevrekidis ${ }^{\text {a }}$, B.A. Malomed ${ }^{\text {b }}$, D.J. Frantzeskakis ${ }^{\text {c }}$, A.R. Bishop ${ }^{\text {, }}$, \\ H.E. Nistazakis ${ }^{\mathrm{c}}$, R. Carretero-González ${ }^{\mathrm{e}, *}$ \\ ${ }^{a}$ Department of Mathematics and Statistics, University of Massachusetts, Amherst, MA 01003-4515, USA \\ ${ }^{\mathrm{b}}$ Department of Interdisciplinary Studies, Faculty of Engineering, Tel Aviv University, Tel Aviv 69978, Israel \\ ${ }^{c}$ Department of Physics, University of Athens, Panepistimiopolis, Zografos, Athens 15784, Greece \\ ${ }^{\mathrm{d}}$ Center for Nonlinear Studies and Theoretical Division, Los Alamos National Laboratory, Los Alamos, NM 87545, USA \\ ${ }^{\mathrm{e}}$ Nonlinear Dynamical Systems Group, Department of Mathematics and Statistics, San Diego State University, \\ 5500 Campanile Drive, San Diego, CA 92182-7720, USA
}

Available online 5 March 2005

\begin{abstract}
We demonstrate the possibility of creating domain walls described by a single component Gross-Pitaevskii equation with attractive interactions, in the presence of an optical-lattice potential. While it is found that the domain wall is unstable in an infinite system, we show that the external magnetic trap can stabilize it. Stable solutions also include "twisted" domain walls, as well as asymmetric solitons. The results apply as well to spatial solitons in planar nonlinear optical waveguides with transverse modulation of the refractive index.
\end{abstract}

(C) 2005 IMACS. Published by Elsevier B.V. All rights reserved.

Keywords: Domain wall; Soliton; Matter waves; Optical lattice; Bose-Einstein condensation

\section{Introduction}

Solitary waves, such as bright and dark solitons, are ubiquitous dynamical patterns in the fields of nonlinear optics and matter waves [1,2]. Besides these customary waveforms, solitary coherent structures in the form of domain walls (DWs) were also predicted in optical fibers with normal group-velocity

\footnotetext{
* Corresponding author. Tel.: +1 619594 7252; fax: +1 6195942029.

E-mail address: carreter@ sciences.sdsu.edu (R. Carretero-González). URL: http://nlds.sdsu.edu (R. Carretero-González).
} 
dispersion carrying two waves with orthogonal circular polarizations, that interact through the crossphase modulation induced by the Kerr nonlinearity $[3,4]$. Similar structures were also predicted in planar nonlinear optical waveguides [5]. The fiber-optic DWs are distinguished by the property that, asymptotically, they contain a single polarization, with a switch between the polarizations in a localized region. Optical-DW solutions were, in fact, found following the pattern of earlier known solutions of the DW (alias "grain-boundary") type in systems of coupled Ginzburg-Landau equations that describe interactions between roll patterns [6,7] or traveling waves [8], with different orientations, in a convection layer.

DWs may also arise in very different physical media (that, however, bear a similar mathematical description), namely, binary (two-component) Bose-Einstein condensates (BECs). In particular, stable DW configurations have been predicted in quasi-one-dimensional (cigar-shaped) binary BECs [9]. Such stable solutions can also exist in the presence of a periodic optical-lattice (OL) potential [10], combined with the parabolic magnetic trap [11].

Optical DWs, as well as periodic DW trains, have been experimentally observed in fibers $[12,13]$. On the other hand, binary BECs have been experimentally created, for instance, as mixtures of different spin states of ${ }^{87} \mathrm{Rb}$ [14], including cases when an OL was used for the confinement [15]. Efforts were also made to create binary BECs with different atomic species, such as ${ }^{41} \mathrm{~K}$ and ${ }^{87} \mathrm{Rb}$ [16], or ${ }^{7} \mathrm{Li}$ and ${ }^{133} \mathrm{Cs}$ [17]. However, DWs have not yet been observed in the experiment, therefore a relevant issue is to predict more favorable conditions for their existence.

Here, we propose a setting in which patterns of the DW type can be realized in a single-component $\mathrm{BEC}$ with attractive interactions between atoms. We demonstrate that this may occur, under suitable conditions, in the presence of the OL potential. Furthermore, we show that the corresponding DW solutions can be stabilized by the magnetic-trap potential. Our results also predict the existence of patterns of the same type in nonlinear-optical media, such as a multichannel nonlinear planar waveguide with transverse modulation of the refractive index [18], or, possibly, in two-dimensional photonic crystals. In particular, the attractive character of the cubic nonlinearity, which is necessary for the existence of the DW, is provided in optics by the usual Kerr effect.

The presentation is structured as follows: in Section 2, we give a detailed formulation of the model and develop an analytical approach to DW solutions, based on the variational approximation (VA). In Section 3, we construct the DW solution numerically, both in the absence and in the presence of the magnetic trap; we obtain a variety of new solutions, including an asymmetric DW-type soliton and a twisted DW, as well as multi-DW patterns. Section 4 concludes the paper.

\section{Formulation of the model and variational approximation}

Assuming that the nonlinear interactions are weak relative to tight confinement in the transverse dimensions, the radial size of the cigar-shaped condensate is much smaller than its length in the axial direction. In such a case, which can be realized in strongly anisotropic traps, the Gross-Pitaevskii equation (GPE), which governs the BEC in the mean-field approximation, assumes an effectively one-dimensional form [19]:

$$
i \psi_{t}=-\psi_{x x}+g|\psi|^{2} \psi+V(x) \psi
$$


where $\psi$ is the single-atom wave function, $t$ and $x$ are measured, respectively, in units of $2 / \omega_{\perp}$ and the transverse harmonic-oscillator length $a_{\perp} \equiv \sqrt{\hbar /\left(m \omega_{\perp}\right)}$ ( $m$ and $\omega_{\perp}$ are the mass and transverse confining frequency), and the energy unit is $\hbar \omega_{\perp} / 2$. The nonlinearity prefactor $g \equiv 2(2 \pi)^{3 / 2} a / a_{\perp}$ is proportional to the scattering length $a$ of the inter-atomic interactions (see, e.g., Refs. [19,20]), $a>0(a<0)$ corresponding to the repulsive (attractive) interactions.

In the general case, the confining potential in Eq. (1) is

$$
V(x)=\Omega^{2} x^{2}+V_{0} \cos (k x),
$$

where the two terms represents the magnetic trap and the OL, respectively. In Eq. (2), $\Omega \equiv \omega_{x} / \omega_{\perp}\left(\omega_{x}\right.$ being the axial confining frequency) is the effective strength of the magnetic trap, $V_{0} \equiv 2 E_{\text {rec }} / \hbar \omega_{\perp}$ is the OL strength $\left(E_{\text {rec }} \equiv h^{2} / 2 m \lambda_{\text {laser }}^{2}\right.$ is the recoil energy, $\lambda_{\text {laser }}$ being the wavelength of the counter-propagating laser beams which generate the OL) and $k$ is the wavenumber of the OL. In the experiment, the spatial period of the OL potential can be controlled not only by changing $\lambda_{\text {laser }}$, but also, more conveniently, by varying the angle $\theta$ between the laser beams, as the local intensity in the interference pattern is modulated at the wavelength $\lambda \equiv 2 \pi / k=\left(\lambda_{\text {laser }} / 2\right) \sin (\theta / 2)[21]$.

To estimate actual physical quantities in this situation, we can take a typical example of the attractive $(a<0)$ condensate of ${ }^{7} \mathrm{Li}$ containing $10^{3}$ atoms, confined in a cigar-shaped trap with the frequencies $\omega_{x}=2 \pi \times 60 \mathrm{~Hz}$ and $\omega_{\perp}=2 \pi \times 400 \mathrm{~Hz}$. This implies $\Omega=0.15 \mathrm{in} \mathrm{Eq.} \mathrm{(2),} \mathrm{while} \mathrm{the} \mathrm{time} \mathrm{and} \mathrm{space} \mathrm{units}$ correspond to $0.8 \mathrm{~ms}$ and $2 \mu \mathrm{m}$, respectively. Finally, concerning the OL wavenumber, $k=2$ (this is a value that will be dealt with in this work) corresponds to the wavelength $\lambda \simeq 6 \mu \mathrm{m}$ of the interference pattern (in physical units). These values can be used for the interpretation of the results that are presented below in terms of the dimensionless variables.

Stationary solutions to Eq. (1) are sought for as $\psi(x, t)=\mathrm{e}^{-\mathrm{i} \mu t} u(x)$, where $\mu$ is the chemical potential (proportional to the energy per atom) and the real function $u$ obeys the equation

$$
\mu u+u^{\prime \prime}-g u^{3}-V(x) u=0,
$$

the prime standing for $\mathrm{d} / \mathrm{d} x$. DW-like solutions are those following the pattern of the ansatz

$$
u(x)=\frac{A}{2}[1-\operatorname{sgn}(x-\xi)],
$$

where $A$ is the amplitude of the wave function at $x=-\infty$ (assuming an infinitely long system) and $x=\xi$ is the location of the DW's center. In linear quantum mechanics, a state of this type cannot exist because the atoms would tunnel from the filled domain, $x<\xi$, into the empty one, $x>\xi$. However, in the GP equation (1), the nonlinear mean-field term adds a negative contribution to the effective potential in the filled domain. As a result, the tunneling will be suppressed, and a DW state of the type (4) is possible, provided that $\mu$ is negative.

Of course, such qualitative considerations provide no guarantee that a DW waveform will indeed exist. To investigate this possibility in quantitative terms, we will first resort to the variational approximation, which will be followed by direct numerical analysis in the next section. To this end, we note that Eq. (3) can be derived from the Lagrangian, $L=\int_{-\infty}^{+\infty} \mathcal{L} \mathrm{d} x$, with the density

$$
\mathcal{L}=u_{x}^{2}-\mu u^{2}+V(x) u^{2}+\frac{g}{2} u^{4} .
$$


For the VA, the potential $V(x)$ does not include the magnetic parabolic trap; see Eq. (2). The effects of including the magnetic trap will be studied numerically (see next section).

With the use of the ansatz (4), the integral, which gives the Lagrangian $L$ diverges, in the infinite system, as $x \rightarrow-\infty$. The divergence may be regularized by setting an edge at $x=-(2 \pi / k) N$ with a large integer $N$ (recall $2 \pi / k$ is the spatial period of the OL), and then taking the limit $N \rightarrow \infty$ (cf. a similar analysis in [23]). This way, it can be easily checked that the diverging part contributes solely to the equation generated by the variation of the Lagrangian in $A$, which is

$$
A^{2}=\frac{\mu}{g}
$$

hence $\mu$ and $g$ should be of the same sign. Because we actually need $\mu<0$ for the existence of the DW (see above), this implies that $g<0$, i.e., the attraction between atoms in BECs (which occurs in ${ }^{7} \mathrm{Li}$ [22], as mentioned above, or in ${ }^{85} \mathrm{Rb}$ [24]), is a necessary condition for the existence of the DW.

The actual shape of the DW differs from the simple ansatz (4) by the presence of the oscillating part in the wave function at $x \rightarrow-\infty$; see Fig. 1(c). In the lowest approximation, treating the OL term in Eq. (3) as a small perturbation, one can find the asymptotic form of the wave function at $x \rightarrow-\infty$ in the form

$$
u_{\text {asympt }}(x)=\sqrt{\frac{\mu}{g}}\left[1-\frac{V_{0}}{2 \mu+k^{2}} \cos (k x)\right]
$$

[the expression (6) was substituted for the amplitude] and the ansatz (4) can be modified accordingly, $u=u_{\text {asympt }}(x)[1-\operatorname{sgn}(x-\xi)]$.

The diverging term in the Lagrangian does not affect the remaining variational equation, $\partial L / \partial \xi=0$ (cf. the situation in the case of solitons with non-vanishing tails, where variational equations for the core of the soliton can also be derived in a divergence-free form [23]). An eventual result is

$$
V_{0} \cos (k \xi)=\mu \frac{2 \mu+k^{2}}{4\left(\mu+k^{2}\right)}
$$

Eq. (8) describes equilibrium between the negative pressure force acting on the DW from the filled domain (the attraction tends to pull the atoms in) and the pinning force induced by the OL. The main conclusion following from Eq. (8) is that a necessary condition for the existence of the DW solution is that the strength of the OL must exceed a minimum (threshold) value

$$
\left|V_{0}\right|_{\mathrm{thr}}=\left|\mu \frac{2 \mu+k^{2}}{4\left(\mu+k^{2}\right)}\right| .
$$

We note that the vanishing of the expression (9) in the case of $2 \mu+k^{2}=0$ is a formal feature, produced by the divergence of the oscillating correction in Eq. (7) in this case. In fact, this is a resonant case, and a proper form of the correction can be derived using well-known methods of nonlinear-resonance theory. We do not consider this special case here.

Comparison results presented in the next section show that the threshold value found from a full numerical solution is smaller than the one given by Eq. (9) by a factor $\sim 8$. This discrepancy may be explained by the assumption, implied in the ansatz (4), that the DW has zero width; in fact, numerical results [see Fig. 1(c)] demonstrate that it is indeed narrow, but still its width is not much smaller than $\lambda=2 \pi / k$. The VA can be extended to incorporate a finite width of the DW; however, the analytical results then become very cumbersome, therefore they are not displayed here. 

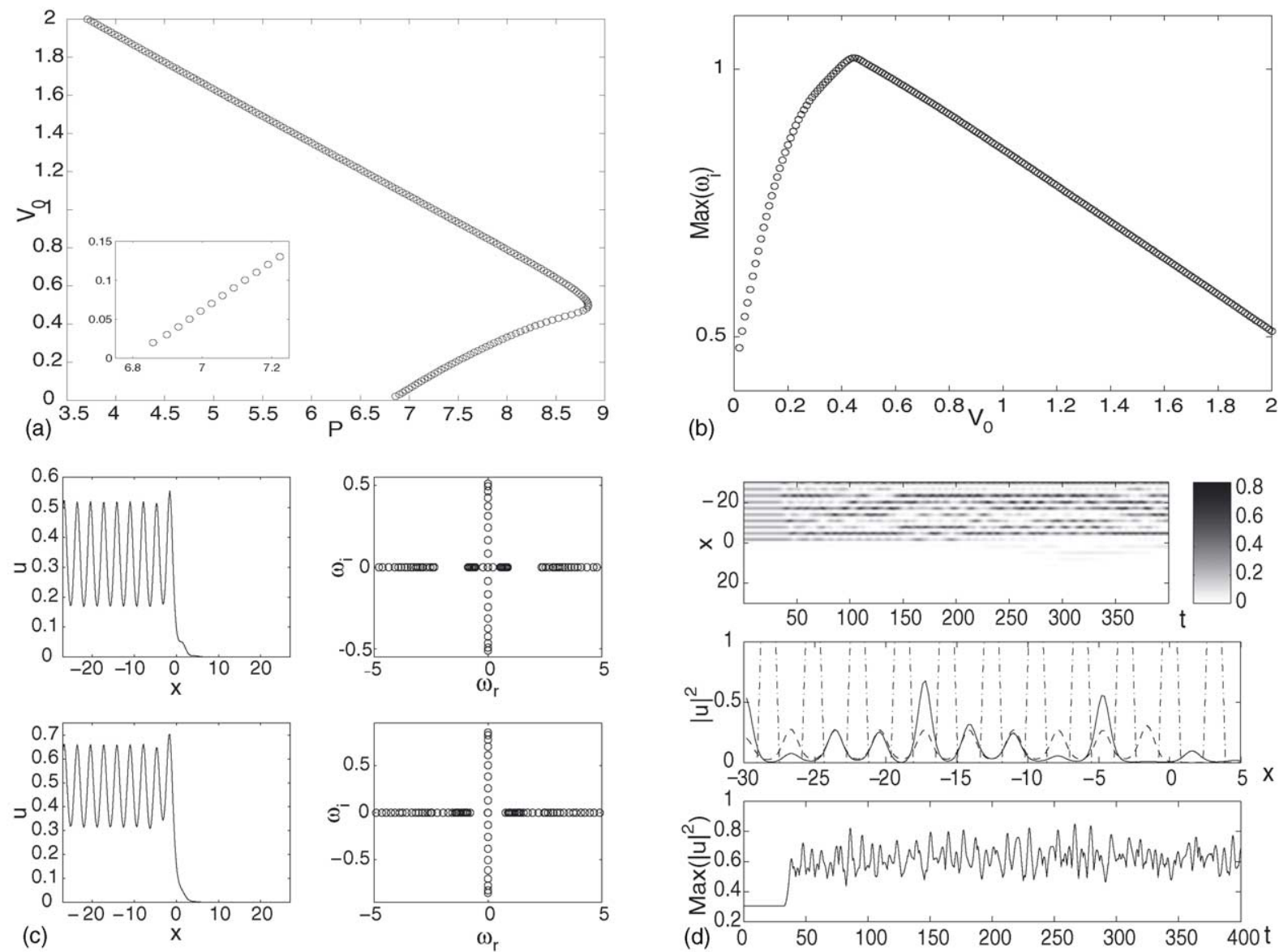

Fig. 1. Domain wall solution in the presence of the external potential $V(x)=V_{0} \cos (2 x)$ (cf. Eq. (2)), namely a pure optical lattice without magnetic trap $\left(\Omega=0\right.$ ). (a) The norm of the domain wall (DW) solution, $P=\int|u|^{2} \mathrm{~d} x$ (alias the normalized number of particles) vs. the optical-lattice's strength $V_{0}$. The inset explicitly shows that the branch starts at a finite threshold value of the strength, $\left|V_{0}\right|_{\text {thr }} \approx 0.02$. (b) The largest instability growth rate (imaginary part of the eigenfrequency) of the DW solution vs. $V_{0}$. This plot shows that the solution is always unstable. (c) The spatial profile of the solution (left subplots) and the corresponding spectral plane of the stability eigenvalue $\left(\omega_{\mathrm{r}}, \omega_{\mathrm{i}}\right)$ (right subplots) for $V_{0}=2$ and 1 (top and bottom subplots, respectively). (d) The space-time contour plot of the density $|u|^{2}$ showing the evolution of the unstable DW in the case of $V_{0}=2$ (top subplot). The middle subplot shows the initial and final configurations (dashed and solid lines), and the optical-lattice potential (dash-dotted line). In order to monitor the instability development, the maximum of the density in the spatial domain is shown, as a function of time, in the bottom subplot.

\section{Numerical results}

We will present results of computations for a typical case within the range of parameters in which the VA predicts the existence of the DW, namely for $g=-3$ and $\mu=-1$. This case can be readily implemented in the experiment, adjusting the magnitude of the scattering length $a$ (which sets the value of the normalized nonlinearity parameter $g$ ) by means of the Feshbach resonance induced by external magnetic field [25] (see also Ref. [26]); for other values of $g$ and $\mu$, the results are quite similar. 
With fixed $g$ and $\mu$ in what follows, we vary the parameters of the potential to obtain different types of solutions.

The stationary Eq. (3) was solved by means of a standard Newton-type algorithm. Once a solution $u_{0}$ to this equation was found, its linear stability was examined, using a straightforward form of the perturbed solution,

$$
\psi(x, t)=\mathrm{e}^{-\mathrm{i} \mu t}\left\{u_{0}(x)+\left[\delta a(x) \exp (-\mathrm{i} \omega t)+\delta b(x) \exp \left(\mathrm{i} \omega^{*} t\right)\right]\right\}
$$

where $(\cdot)^{*}$ denotes the complex conjugation. The resulting linear equations for the small perturbations $\delta a$ and $\delta b$ are

$$
\left\{\begin{array}{l}
\omega \delta a=-(\delta a)^{\prime \prime}+V(x) \delta a+2 g u_{0}^{2} \delta a-\mu \delta a+g u_{0}^{2}(\delta b)^{*} \\
\omega^{*} \delta b=(\delta b)^{\prime \prime}+V(x) \delta b-2 g u_{0}^{2} \delta b+\mu \delta b-g u_{0}^{2}(\delta a)^{*}
\end{array} .\right.
$$

These can be tackled by means of a matrix eigenvalue solver (using the finite-difference discretization with a spatial step chosen to be $\Delta x=0.2$ ). The eigenfrequencies $\omega$ thus found will be shown in the spectral plane $\left(\omega_{\mathrm{r}}, \omega_{\mathrm{i}}\right)$, where the subscripts denote their real and imaginary parts. The configuration is linearly unstable if there is an eigenfrequency with a non-vanishing imaginary part $\omega_{\mathrm{i}} \neq 0$.

If the stationary solution was found to be unstable, a fourth-order Runge-Kutta integrator, with time step $\Delta t=0.001$ and no-flux boundary conditions at the edges of the domain, was used to directly simulate the development of the instability.

Numerical results for the stationary DW solutions are displayed in Fig. 1. In this case, we use the OL potential with $k=2$ without the parabolic trap [ $\Omega=0$ in Eq. (2)] and examine the behavior of the DW as a function of the potential's strength $V_{0}$. The solutions indeed exist only if $V_{0}$ exceeds a finite threshold value $\left|V_{0}\right|_{\text {thr }} \approx 0.02$, which is smaller than the one (9) predicted by the VA in the crude approximation. A reason for the discrepancy was discussed above, however, the most important feature of the VA is that it successfully predicts the existence of the finite threshold.

Fig. 1 shows that this family of solutions is unstable for every value of $V_{0}$; see an explanation below. An example of the instability development included in Fig. 1 (for $V_{0}=2$ ) shows that, on a relatively short time scale ( $\sim 30$ in this case), the instability destroys the DW configuration, leading to an apparently chaotic redistribution of the density between different potential wells and gradual expansion of the condensate into the originally empty domain.

The next step is to consider the effect exerted on DWs by the external magnetic (parabolic) trapping potential in the system $(\Omega \neq 0)$, which is typically an inherent ingredient of experimental BEC settings [20]. The trap may partly stabilize the DW, by reducing the number of unstable eigenvalues, as illustrated by Fig. 2, where the combined optical and magnetic potential is imposed, with $\Omega^{2}=0.01, V_{0}=2$ and $k=2$. Notice, more generally, that the presence of the magnetic trap will result in fewer humps of the DW (for larger values of the trapping frequency $\Omega$, hence smaller values of the Thomas-Fermi radius $R_{\mathrm{TF}}=\sqrt{|\mu| / \Omega}$ ). There is a competition between the length scale of the optical potential $2 \pi / k$ and the one of the magnetic trapping, which will determine how many humps can "fit" in a DW, in their combined presence. In this case, it is seen that the trap confines the pattern to three humps in half of the region $|x|<R_{\mathrm{TF}}\left(R_{\mathrm{TF}} \approx 10\right.$ in this case; the presence of the $\mathrm{DW}$ is evident from the fact that the interval $0<x<R_{\mathrm{TF}}$ is empty). This pattern is still unstable and its evolution results in a structure that alternates between a large single-well pulse and a double-humped one trapped in two adjacent wells. 

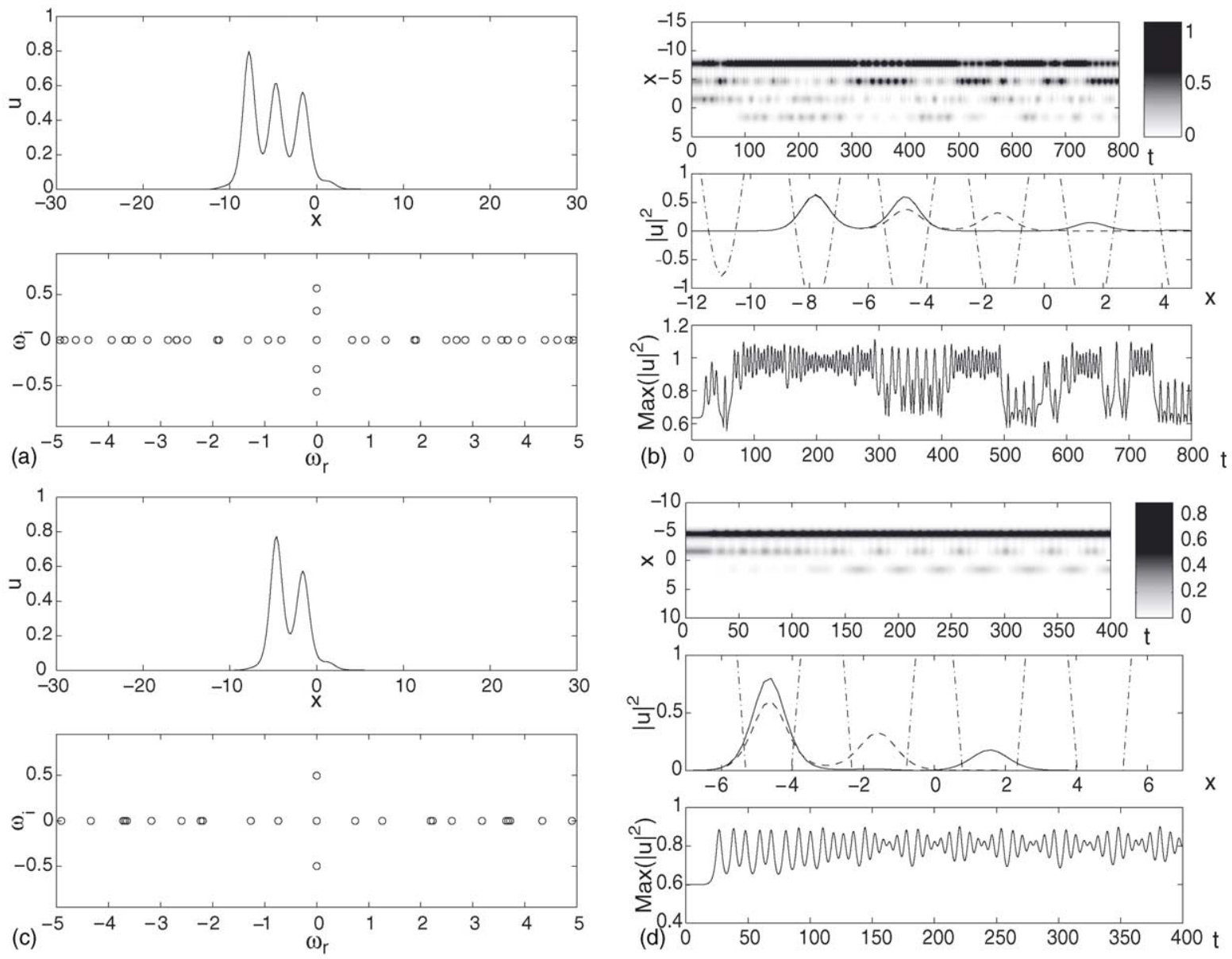

Fig. 2. (a) The profile and spectral plane of a three-humped DW found in the potential $V(x)=0.01 x^{2}+2 \cos (2 x)$. (b) The instability development of the pattern. Generally, it tends to collect the condensate in a single potential well. However, there are also time intervals in which the atoms form two pulses in two adjacent wells (the pulses have opposite signs). (c and d) The same as in (a) and (b), for a double-humped DW in the potential $V(x)=0.025 x^{2}+2 \cos (2 x)$.

The stability properties of the three-humped DW configuration considered above shed light on the origin of the instability of the DWs. In particular, we observe in the bottom subplot of Fig. 2(a) that this structure has two unstable eigenfrequency pairs, which is to be compared with the right part of Fig. 1(c), where, in the absence of the magnetic trap, many unstable eigenfrequencies are present. In this connection, we note that, by means of the substitution $\delta a=w+v, \delta b=w^{*}-v^{*}$, Eq. (11) can be re-written in the form of

$$
\left\{\begin{array}{l}
\omega v=-w^{\prime \prime}+V(x) w+3 g u_{0}^{2} w-\mu w \equiv-L_{+} w \\
\omega w=-v^{\prime \prime}+V(x) v+g u_{0}^{2} v-\mu v \equiv-L_{-} v
\end{array} .\right.
$$

The operator $L_{-}$has $u_{0}$ as its zero mode, i.e., an eigenstate with $\omega=0$. Hence, it follows from Sturm-Liouville theory that, since $u_{0}$ does not change its sign, the number of negative eigenvalues 
of $L_{-}$is $n\left(L_{-}\right)=0$. On the other hand, $u_{0}^{\prime}$, the spatial derivative of $u_{0}$, is an $\omega=0$ eigenstate of the operator $L_{+}$(in the absence of external potential). Again, using Sturm-Liouville theory, it can be deduced (cf. also [27]) that the number of negative eigenvalues of $L_{+}$(if $u_{0}$ contains $N$ separated humps) is $n\left(L_{+}\right)=N$. Consequently, $\left|n\left(L_{+}\right)-n\left(L_{-}\right)\right|=N$. One can then infer from the arguments of Refs. [28,29] that there must exist $N-1$ unstable real eigenvalue pairs (imaginary eigenfrequency pairs) in such a case. This clearly explains the existence of the two unstable eigenvalue pairs in the case of the three-humped DW.

The above arguments are also supported by panels (c) and (d) of Fig. 2: here, for a more tightly confining magnetic trap, with $\Omega^{2}=0.025$ (the OL parameters are again $V_{0}=2$ and $k=2$ ), the resulting configuration is a double-humped DW (the larger the magnetic-trap strength $\Omega$, the smaller the Thomas-Fermi radius $R_{\mathrm{TF}}$, hence, the number of the "humps" decreases). In this case, $N=2$, and therefore, in agreement with the above arguments, only one unstable eigenfrequency can be identified in the spectral plane $\left(\omega_{\mathrm{r}}, \omega_{\mathrm{i}}\right)$; see bottom subplot in Fig. 2(c). Fig. 2(d) shows that the instability leads to absorption of a large fraction of the atoms from one "hump" by the other. However, a small fraction of atoms tunnels to the next well. We have verified that the small pulse generated in this well has parity opposite to that of the large one on which it abuts. Such opposite-parity configurations may be stable, see below.

Previous results for multi-pulse configurations in discrete nonlinear-Schrödinger models with attractive interaction [27,30-35] have shown that, while patterns composed of same-parity pulses (i.e., ones with no zero-crossings between them) are always unstable, the so-called twisted localized modes (TLMs), in which adjacent pulses are of opposite parity, may be stable. This can be seen, in the context of the above consideration, from the fact that if there are $S$ zero-crossings in the profile $u_{0}$, then $n\left(L_{-}\right)=S$ and hence, the Jones-Grillakis criterion [28,29] predicts $N-S-1$ real-eigenvalue (imaginary-eigenfrequency) pairs. Thus, if $S=N-1$ (i.e., if adjacent pulses have opposite parities), then the solution is potentially stable, although other types of instabilities are also known to occur in this case [31-35], such as the oscillatory instability (accounted for by a Hamiltonian Hopf bifurcation) [36] due to a negative eigendirection in the energy of small perturbations $[37,38]$.

Following this argument, we looked for a twisted-DW configuration, which we were indeed able to identify, as shown in Fig. 3. To examine the existence and stability of this type of patterns, we used continuation in the OL strength $V_{0}$, and found that it exists for $V_{0} \geq 0.25$; see the top part of Fig. 3(a). Furthermore, it is unstable, due to the above-mentioned oscillatory instability, in the intervals $0.25<V_{0} \leq 0.32$ (i.e., just after it emerges) and $0.41 \leq V_{0} \leq 0.53$, while it is stable otherwise; see bottom subplot of Fig. 3(a). In the case of the instability, we simulated its evolution, as shown in Fig. 3(c). It is observed that the instability cleaves the twisted DW through an oscillatory perturbation [see the top and bottom subplots of panel (c) in the interval $175 \leq t \leq 250$ ] and results in oscillations around a configuration with most of the atoms trapped in a single well. This configuration is actually an asymmetric soliton (see also below).

The latter result, as well as the natural expectation that, if the Thomas-Fermi radius $R_{\mathrm{TF}}$ becomes sufficiently small, a single-humped DW pattern may emerge, which would be a new type of an asymmetric solitary wave in the present context, led us to search for a single-humped DW. Such a solution has been found, and is shown in Fig. 4 for different values of $V_{0}$ in the potential with $\Omega^{2}=0.06$ and $k=2$. It is noteworthy that the solution is stable for all the examined values of $V_{0}$. For a small OL strength, the solution degenerates into the regular symmetric soliton, which is possible in the one-dimensional GP equation with attractive interactions, and has been observed in the experiment [39].

Finally, we examined possibilities to create composite DWs, constructed as bound states of the regular or twisted fundamental DWs, which were considered above. Such configurations have been found. Two 

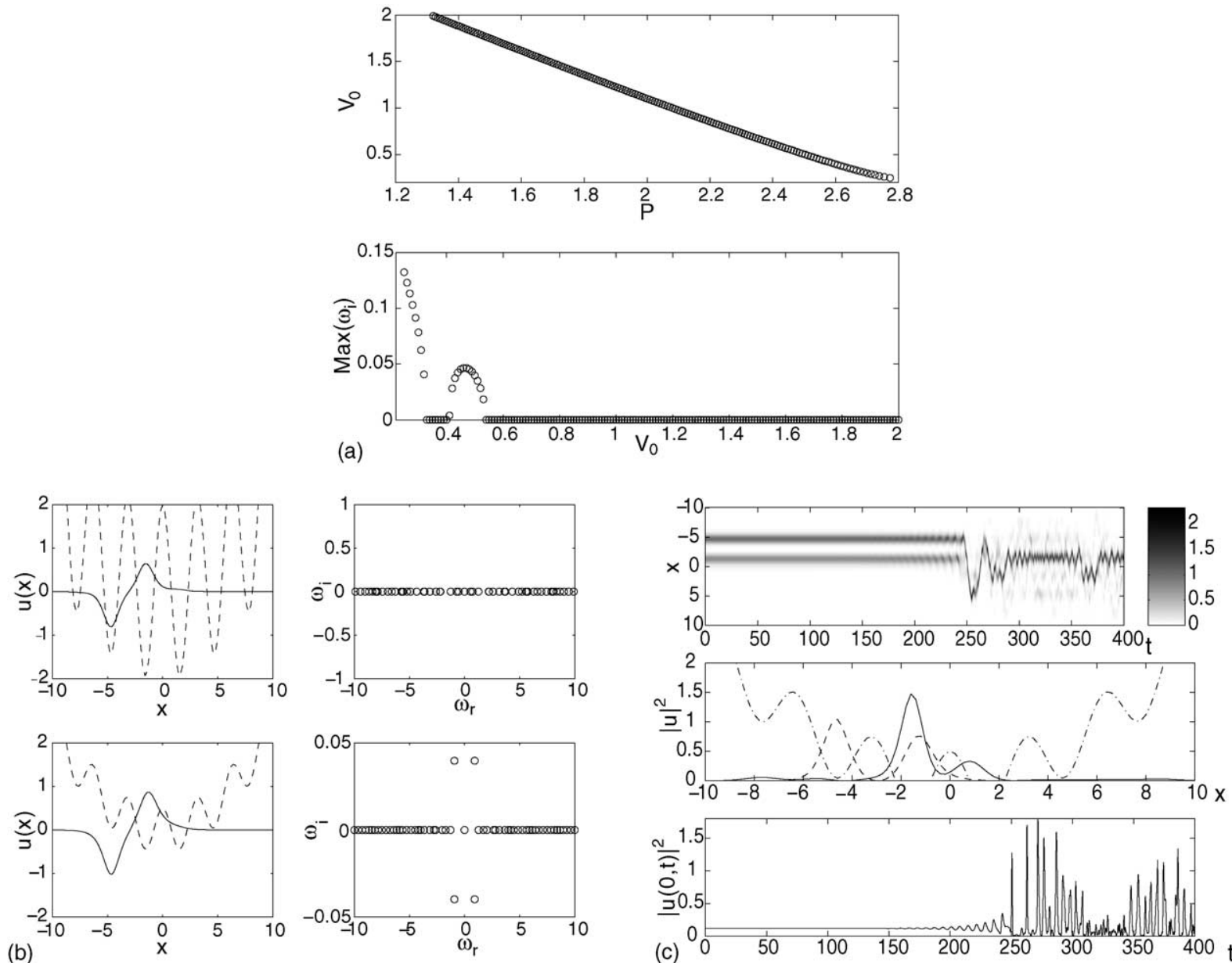

Fig. 3. (a) The branch of the twisted-DW solutions. The bottom subplot of the panel shows the most unstable eigenvalue of small perturbations around the configuration [the instability, if any, has an oscillatory character; see panel (c)]. (b) Examples of the twisted DW for $V_{0}=2$ and 0.5 (top and bottom subplots; the dashed line indicates the potential) and their linear stability spectra. (c) The evolution of an unstable twisted DW, which results in transition to an asymmetric single-humped soliton. Panel (c) is similar to Fig. 1(d) except for the bottom part illustrating the instability development through the time evolution of the condensate density at the bottom of the magnetic trap, $|u(0, t)|^{2}$.

typical examples, viz., bound states of double-humped DWs for $\Omega^{2}=0.025, V_{0}=2.5$ and $k=2$, and of twisted DWs for the same $\Omega^{2}$ and $k$ but for $V_{0}=1.9$, are shown in Fig. 5. Naturally, as the former configuration has one pair of unstable eigenvalues per each double-humped DW, the composite profile bears two such pairs; similarly, since each single twisted DW may be stable, their bound state may be stable too (but it may also be subject to the oscillatory instability shown above). The unstable evolution of the bound state of the double-humped DWs from the top subplot of Fig. 5(a) is shown in panel (b) of the figure. Clearly, each of the constituent DWs is destroyed in favor of a twisted-DW pattern, in which the constituent pulses are far separated. 

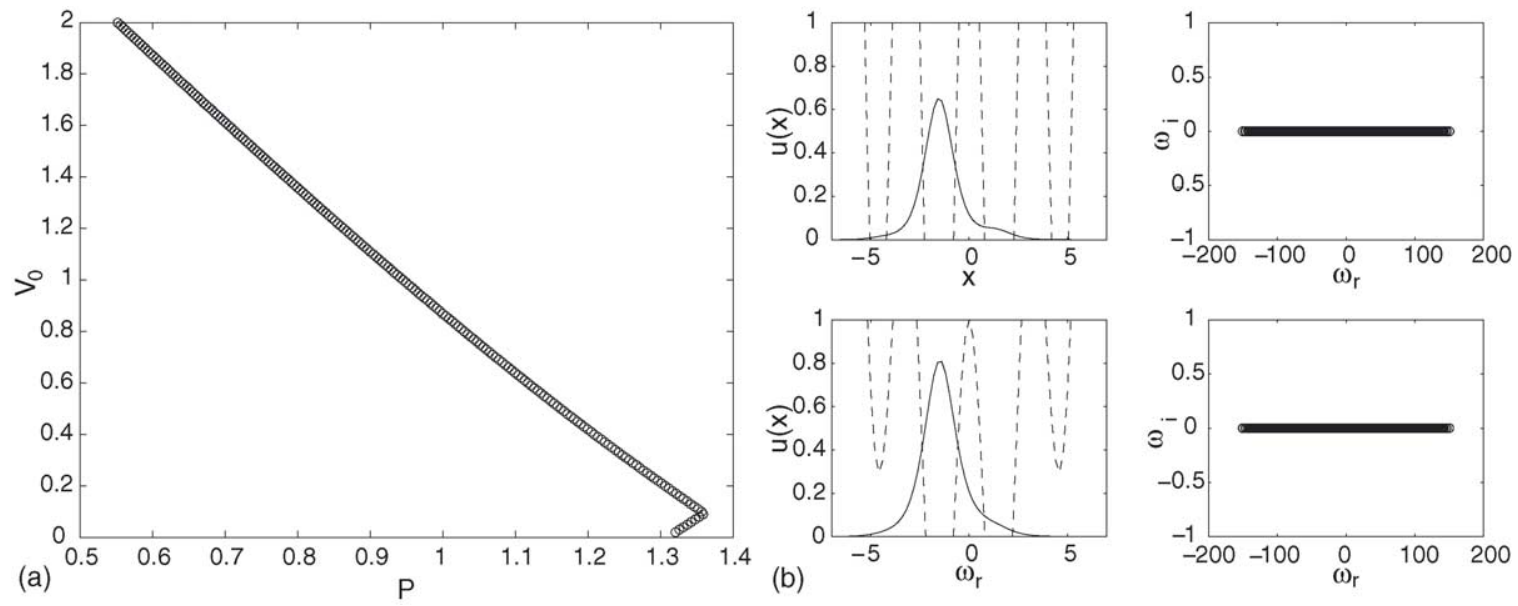

Fig. 4. (a) The branch of asymmetric-DW solutions and (b) profiles and stability of the solutions for $V_{0}=2$ and 1 (top and bottom subplots).
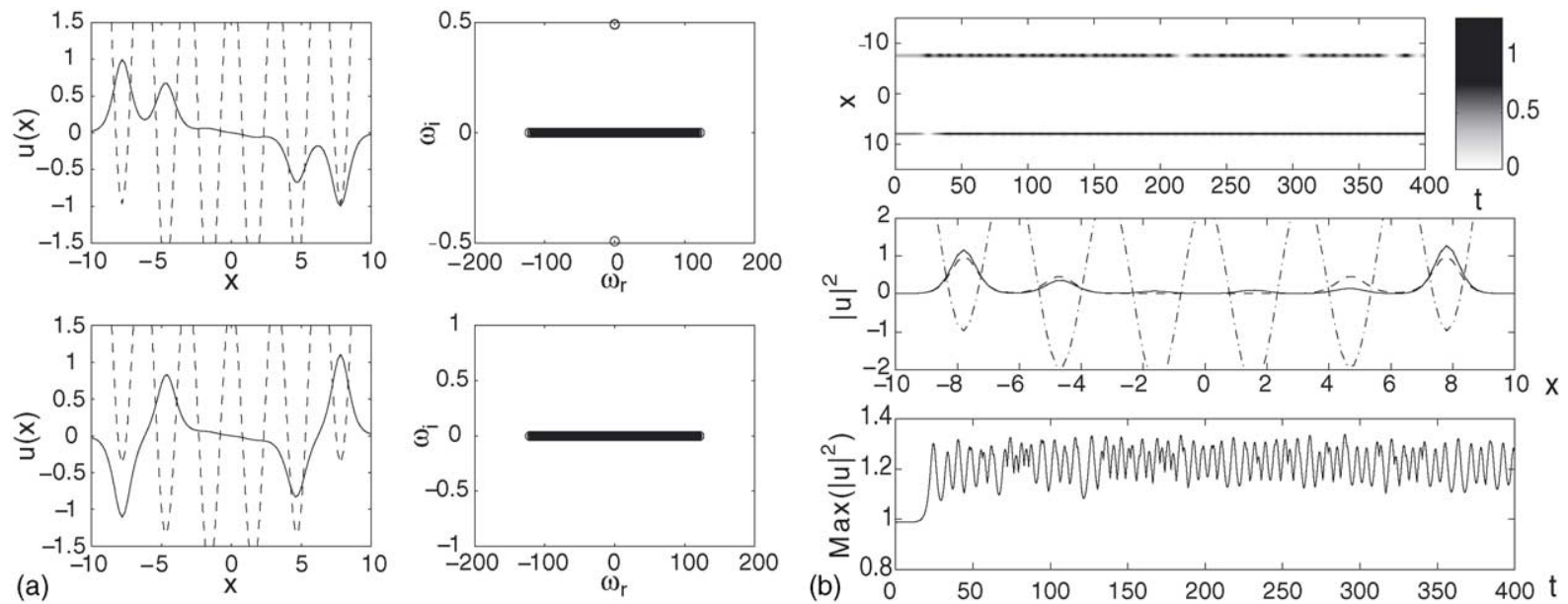

Fig. 5. (a) The bound state of double-humped DWs for $V(x)=0.025 x^{2}+2.5 \cos (2 x)$ (shown by the dashed line) and its stability (right subplot). The configuration has two nearly identical unstable eigenvalues. The bottom subplot shows a stable bound state of twisted DWs for $V(x)=0.025 x^{2}+1.9 \cos (2 x)$. (b) The development of the instability of the bound state of double-humped DWs from the top subplot of (a) leading to the formation of two larger-amplitude out-of phase pulses. The panel (b) displays similar features as Fig. 1(d) for the double-humped DW.

\section{Conclusions}

Domain wall solutions appear in a variety of contexts in optics and matter-wave physics, in both theoretical and experimental studies (experimentally, DWs have been observed in nonlinear optical fibers). Most of these settings are inherently two-component ones with immiscible components, the repulsion between them making it possible to support stable DWs. 
In this work, we have investigated possibilities to create domain walls in single-component models (particularly, in those based on the Gross-Pitaevskii equation, and relevant to the description of Bose-Einstein condensates) in the presence of external potentials (OL and parabolic trap). The same model is relevant to the study of spatial solitons in planar waveguides with transverse modulation of the refractive index. By means of the analytical approach, based on variational methods, and through direct simulations we have found that DWs are possible only in the model with intrinsic attraction, and a necessary condition is that the OL strength must exceed a threshold value. Regular domain walls (with no zero-crossings) in the OL potential are found to be unstable, for which an explanation was given. If the magnetic trap is introduced, twisted DWs can be stable, as well as asymmetric single-pulse soliton solutions. Bound states of elementary DWs (regular and twisted ones) have also been investigated; they have a chance to be stable in the latter case.

A natural extension of the present study is to search for multi-dimensional counterparts of these solutions; these results will be reported elsewhere.

\section{Acknowledgements}

P.G.K. gratefully acknowledges support from NSF-DMS-0204585 and from the Eppley Foundation for Research. Work at Los Alamos is supported by the US DoE. B.A.M. acknowledges a partial support from the Israel Science Foundation through a Research-Excellence-Center Grant No. 8006/03.

\section{References}

[1] G.P. Agrawal, Applications of Nonlinear Fiber Optics, Academic Press, San Diego, 2001.

[2] Yu.S. Kivshar, G.P. Agrawal, Optical Solitons, Academic Press, Amsterdam, 2003.

[3] S. Wabnitz, B. Daino, Phys. Lett. A 182 (1993) 289.

[4] B.A. Malomed, Phys. Rev. E 50 (1994) 1565.

[5] M. Haelterman, A. Sheppard, Phys. Lett. A 185 (1994) 265.

[6] B.A. Malomed, A.A. Nepomnyashchy, M.I. Tribelsky, Phys. Rev. A 42 (1990) 7244.

[7] A. Hari, A.A. Nepomnyashchy, Phys. Rev. E 50 (1994) 1661;

A. Hari, A.A. Nepomnyashchy, Phys. Rev. E 61 (2000) 4835.

[8] B.A. Malomed, Phys. Rev. E 50 (1994) R3310; H. Sakaguchi, B.A. Malomed, Physica D 118 (1998) 250.

[9] M. Trippenbach, K. Goral, K. Rzazewski, B. Malomed, Y.B. Band, J. Phys. B: At. Mol. Opt. 33 (2000) 4017; S. Coen, M. Haelterman, Phys. Rev. Lett. 87 (2001) 140401; P. Öhberg, L. Santos, Phys. Rev. Lett. 86 (2001) 2918.

[10] D.-I. Choi, Q. Niu, Phys. Rev. Lett. 82 (1999) 2022;

A.V. Taichenachev, A.M. Tumaikin, V.I. Yudin, J. Opt. B: Quant. Semicl. 1 (1999) 557;

P. Pedri, L. Pitaevskii, S. Stringari, C. Fort, S. Burger, F.S. Cataliotti, P. Maddaloni, F. Minardi, M. Inguscio, Phys. Rev. Lett. 87 (2001) 220401;

H. Pu, W. Zhang, P. Meystre, Phys. Rev. Lett. 87 (2001) 140405.

[11] P.G. Kevrekidis, H.E. Nistazakis, D.J. Frantzeskakis, B.A. Malomed, R. Carretero-González, Eur. Phys. J. D: At. Mol. Opt. Phys. 28 (2004) 181.

[12] S. Pitois, G. Millot, S. Wabnitz, Phys. Rev. Lett. 81 (1998) 1409.

[13] J.M. Dudley, F. Gutty, S. Pitois, G. Millot, IEEE J. Quant. Electron. 17 (2001) 587.

[14] C.J. Myatt, E.A. Burt, R.W. Ghrist, E.A. Cornell, C.E. Wieman, Phys. Rev. Lett. 78 (1997) 586. 
[15] D.S. Hall, M.R. Matthews, J.R. Ensher, C.E. Wieman, E.A. Cornell, Phys. Rev. Lett. 81 (1998) 1539;

D.M. Stamper-Kurn, M.R. Andrews, A.P. Chikkatur, S. Inouye, H.-J. Miesner, J. Stenger, W. Ketterle, Phys. Rev. Lett. 80 (1998) 2027.

[16] G. Modugno, G. Ferrari, G. Roati, R.J. Brecha, A. Simoni, M. Inguscio, Science 294 (2001) 1320.

[17] M. Mudrich, S. Kraft, K. Singer, R. Grimm, A. Mosk, M. Weidemüller, Phys. Rev. Lett. 88 (2002) 253001.

[18] B.A. Malomed, Z.H. Wang, P.L. Chu, G.D. Peng, J. Opt. Soc. Am. B 16 (1999) 1197.

[19] V.M. Pérez-García, H. Michinel, H. Herrero, Phys. Rev. A 57 (1998) 3837;

L. Salasnich, A. Parola, L. Reatto, Phys. Rev. A 65 (2002) 043614;

Y.B. Band, I. Towers, B.A. Malomed, Phys. Rev. A 67 (2003) 023602.

[20] F. Dalfovo, S. Giorgini, L.P. Pitaevskii, S. Stringari, Rev. Mod. Phys. 71 (1999) 463.

[21] O. Morsch, E. Arimondo, in: T. Dauxois, S. Ruffo, E. Arimondo, M. Wilkens (Eds.), Dynamics and Thermodynamics of Systems with Long-Range Interactions, Springer, Berlin, 2002, pp. 312-331.

[22] A.J. Moerdijk, W.C. Stwalley, R.G. Hulet, B.J. Verhaar, Phys. Rev. Lett. 72 (1994) 40.

[23] D.J. Kaup, B.A. Malomed, Physica D 184 (2003) 153.

[24] J.P. Burke, J.L. Bohn, B.D. Esry, C.H. Greene, Phys. Rev. Lett. 80 (1998) 2097.

[25] S. Inouye, M.R. Andrews, J. Stenger, H.-J. Miesner, D.M. Stamper-Kurn, W. Ketterle, Nat. (Lond.) 392 (1998) 151; E.A. Donley, N.R. Claussen, S.L. Cornish, J.L. Roberts, E.A. Cornell, C.E. Wieman, Nat. (Lond.) 412 (2001) 295.

[26] P.G. Kevrekidis, G. Theocharis, D.J. Frantzeskakis, B.A. Malomed, Phys. Rev. Lett. 90 (2003) 230401.

[27] T. Kapitula, P.G. Kevrekidis, B.A. Malomed, Phys. Rev. E 63 (2003) 036604.

[28] C.K.R.T. Jones, Ergodic Theory Dynamical Syst. 8 (1988) 119-138.

[29] M. Grillakis, Commun. Pure Appl. Math. 46 (1988) 747; M. Grillakis, Commun. Pure Appl. Math. 43 (1990) 299;

M. Grillakis, J. Shatah, W. Strauss, J. Funct. Anal. 74 (1987) 160.

[30] S. Darmanyan, A. Kobyakov, F. Lederer, Sov. Phys. JETP 86 (1998) 682.

[31] P.G. Kevrekidis, A.R. Bishop, K.Ø. Rasmussen, Phys. Rev. E 63 (2001) 036603.

[32] P.G. Kevrekidis, Phys. Rev. E 64 (2001) 026610.

[33] P.G. Kevrekidis, M.I. Weinstein, Math. Comp. Simul. 62 (2003) 65-78.

[34] P.J.Y. Louis, E.A. Ostrovskaya, C.M. Savage, Yu.S. Kivshar, Phys. Rev. A 67 (2003) 013602.

[35] P.G. Kevrekidis, D.J. Frantzeskakis, B.A. Malomed, A.R. Bishop, I.G. Kevrekidis, New J. Phys. 5 (2003) 64.

[36] J.-C. van der Meer, Nonlinearity 3 (1990) 1041.

[37] Dmitry V. Skryabin, Phys. Rev. E 64 (2001) 055601.

[38] T. Kapitula, P.G. Kevrekidis, B. Sandstede, Counting eigenvalues via the Krein signature in infinite dimensional Hamiltonian systems (preprint).

[39] K.E. Strecker, G.B. Partridge, A.G. Truscott, R.G. Hulet, Nature 417 (2002) 150;

L. Khaykovich, F. Schreck, G. Ferrari, T. Bourdel, J. Cubizolles, L.D. Carr, Y. Castin, C. Salomon, Science 296 (2002) 1290 . 